\title{
Annette Höhmann-Kost (Hrsg.): Integrative Bewegungstherapie (IBT). Theorie und Praxis
}

\author{
3. aktualisierte und ergänzte Auflage, Hogrefe, 2018, \\ Bern / Göttingen, 235 Seiten, 29,95€
}

W

enn Autorlnnen sich anschicken, die komplexe Materie der Integrativen Leibund Bewegungstherapie (IBT) in eine verständliche und dennoch nicht zu sehr verkürzende Sprache zu fassen, dann ist dies ein schwieriges Unterfangen. Der Autorin, die die IBT als Therapeutin und Lehrtherapeutin seit vielen Jahren im In- und Ausland vermittelt, ist dies in außerordentlicher Qualität gelungen. Damit ist das Buch hervorragend für Einsteiger in die Integrative Bewegungstherapie geeignet, kann aber auch erfahrenen BewegungstherapeutInnen Anregungen geben, die komplexen Inhalte der IBT in verständlicher Weise ihren PatientInnen und KlientInnen zu vermitteln.

Das Buch wurde in der jetzt erschienenen 3. Auflage aktualisiert und mit ergänzenden Beiträgen versehen. Dies zeigt, dass die Integrative Leib- und Bewegungstherapie ständig in Entwicklung ist und dass sich die Autorin immer wieder auch kritisch mit neuen Entwicklungen auseinandersetzt. Sie versteht Bewegungstherapie dabei als eine psychophysische Methode zur Behandlung und Persönlichkeitsentwicklung, die sozial-ökologische Aspekte einbezieht. Das heißt, sie beschreibt, wie Bewegungstherapie differenziert eingesetzt werden kann, um auf unterschiedlichen Ebenen des menschlichen Daseins Veränderungsprozesse zu initiieren: körperliche, seelische, Prozesse im sozialen Miteinander und im Hinblick auf unser Eingebunden-Sein in unsere Umwelt.

Neben dem Menschenbild der Integrativen Leib- und Bewegungstherapie beschreibt Annette Höhmann-Kost zunächst Kernbegriffe, die das komplexe Verständnis von Bewegungstherapie erläutern. Interessant ist dabei, dass ein Kapitel auch der Bedeutung von Sprache gewidmet ist. Denn Sprache schafft nicht nur Wirklichkeit, sondern auch Atmosphären, die im therapeutischen Prozess von nicht zu unterschätzender Bedeutung sein können.

Auch die Wahrnehmung wird als ein zentrales Anliegen der IBT beschrieben, da sie die Grundlage für zukünftiges Handeln bildet. Über die Wahrnehmung von Muskelspannung, von grundsätzlichen Haltungs- und Bewegungsmustern wie Atmung, Stehen, Sitzen, Gehen und Laufen können Menschen einen Weg zu sich selbst und zu ihren Themen und Problemen entdecken. Die Autorin beschreibt, wie die Begleitung auf diesem Wege in einer behutsamen und feinsinnigen sowie für die PatientInnen fruchtbaren Art und Weise erfolgen kann. Hilfreich ist hier auch eine kleine Übungssammlung als Anregung für die praktische Arbeit.

Ergänzt wird das Buch durch Beiträge zur Bewegungstherapie in der Natur, zur Arbeit mit aggressiven Impulsen und schwierigen Gefühlen, zum Einsatz von Kampfkünsten in der Bewegungstherapie (Budōtherapie) und zum Thema Selbstregulation in der Arbeit mit jungen Erwachsenen.

Alles in allem bietet das Buch eine Essenz langjähriger bewegungstherapeutischer Praxis sowohl der Herausgeberin als auch der CoAutoren, Frank Siegele und Martin Waibel.

\section{Dr. Hermann Ludwig}

DOI 10.2378/ktb2018.art18d 